\title{
Towards an iterative exploration of novel materials exhibiting electronic phase transitions
}

\author{
J.M. Rondinelli \\ Department of Materials Science and Engineering, Northwestern University, Evanston, IL USA \\ jrondinelli@northwestern.edu
}

Over the recent decade, functional electronic materials design and discovery have shifted way from chemical-intuition-based towards data-driven synthesis and simulation. Numerous machine learning models have been developed to successfully predict materials properties and generate new crystal structures. Most existing approaches, however, rely much upon physical insights to construct handcrafted features (descriptors), which are not always readily available. For novel materials with sparse prior data and insufficient physical understanding, conventional machine learning models display limited predictability. In this talk, I will address this challenge by introducing an adaptive optimization engine for materials composition optimization, where feature engineering is not explicitly required. I then describe a use case where multi-objective Bayesian optimization with latent-variable Gaussian processes is utilized to accelerate the design of electronic metal-insulator transition compounds [1]. Next, I will present a quantitative study on the structureproperty relationship in crystal systems enabled by deep neural networks. The model, which learns the structural genome, could identify intrinsically similar structures in Fourier space. Finally, I propose that these two methods could work harmoniously with each other towards an iterative exploration of novel functional materials.

[1] Wang, Y., Iyer, A., Chen, W. \& Rondinelli, J.M. (2020). Appl. Phys. Rev. 7, 041403.

Keywords: metal-insulator transition; lacunar spinel; DFT; machine learning

This work was supported in part by the National Science Foundation under Award No. DMR-1729303. The information, data, or work presented herein was also funded in part by the Advanced Research Projects Agency-Energy (ARPA-E), U.S. Department of Energy, under Award No. DE-AR0001209. The views and opinions of authors expressed herein do not necessarily state or reflect those of the United States Government or any agency thereof. 